\title{
Avaliação Subjetiva Global Produzida pelo Próprio Paciente e Indicadores de Risco Nutricional no Paciente Oncológico em Quimioterapia Patient-Generated Subjective Global Assessment and Nutritional Risk Indicators in Oncology Patients Receiving Chemotherapy Valoración Global Subjetiva Generada por el Paciente e Indicadores de Riesgo Nutricionales en el Paciente con Cancer que Reciben Quimioterapia
}

\author{
Nayara de Souza Gomes'; Regiane Maio $^{2}$
}

\section{Resumo}

Introduçáo: A Avaliação Subjetiva Global Produzida pelo Próprio Paciente é recomendada durante a assistência nutricional em oncologia para detecção precoce do risco nutricional ou desnutrição. A localização do tumor e o esquema quimioterápico podem por si só auxiliar na deteç̧ão do risco nutricional. Objetivo: Descrever a classificação do estado nutricional, segundo método subjetivo, e os indicadores de risco nutricional no paciente oncológico em quimioterapia. Método: Estudo descritivo do tipo série de casos, realizado no setor de quimioterapia de um hospital universitário, de agosto a dezembro de 2014. Participaram da pesquisa indivíduos adultos e idosos, de ambos os sexos, com idade $\geq 20$ anos, diagnosticados com câncer, acompanhados ambulatorialmente e que haviam iniciado o primeiro ciclo de quimioterapia. A localização do tumor e a terapia antineoplásica foram utilizadas como indicadores de risco nutricional. A avaliação nutricional foi realizada pela Avaliação Subjetiva Global Produzida pelo Próprio Paciente. Resultados: Foram estudados 30 pacientes, com idade média de 49,1 $\pm 16,4$ anos. Vinte e cinco entrevistados (83\%) eram tratados exclusivamente com quimioterapia. Segundo a localização do tumor e o esquema quimioterápico, $67 \%$ e $100 \%$, respectivamente, foram considerados de médio a alto risco nutricional. Pela avaliação subjetiva, 17 (56,7\%) apresentaram risco nutricional ou desnutriçáo moderada (grau B) e seis (20\%) desnutrição grave (grau C). Pela pontuação obtida no formulário subjetivo, a necessidade de intervenção nutricional foi verificada em 23 (76,7\%). Conclusáo: $\mathrm{Na}$ amostra estudada, foram elevadas as frequências de tumores e terapias agressivas de risco nutricional/ desnutrição, assim como de necessidade de indicação de terapia nutricional.

Palavras-chave: Avaliação Nutricional; Estado Nutricional; Quimioterapia; Efeitos Colaterais e Reações Adversas Relacionados a Medicamentos; Neoplasias

Trabalho realizado no Setor de Quimioterapia do Hospital das Clínicas da Universidade Federal de Pernambuco (HC/UFPE). Recife (PE), Brasil.

${ }^{1}$ Nutricionista. Especialista em Nutriçấo Clínica pelo Programa de Residência em Nutrição (PRN)/HC/UFPE. Recife (PE), Brasil. E-mail: nayaranut@gmail.com.

${ }^{2}$ Nutricionista. Doutora. Professora-Adjunta da UFPE. Recife (PE), Brasil. E-mail: regmaio@yahoo.com.br.

Enderȩ̧o para correspondência: Nayara de Souza Gomes. Rua Padre Macedo, 233 - Bom Futuro. Fortaleza (CE), Brasil. CEP: 60416-390. E-mail: nayaranut@gmail.com. 


\section{INTRODUÇÃO}

O câncer afeta, de modo crescente, a população mundial. Para o ano de 2030, espera-se que o número de casos novos pela doença oncológica supere os 21,4 milhōes; e o número de óbitos seja de 13,2 milhóes. No Brasil, as estimativas do Instituto Nacional de Câncer José Alencar Gomes da Silva (INCA) apontam para a ocorrência de 576 mil novos casos no país em $2015^{1}$.

A desnutrição é um fator frequentemente encontrado no paciente oncológico e pode ser consequência do aumento da demanda nutricional do tumor, das alteraçóes metabólicas causadas pela enfermidade neoplásica e pelo tratamento oncoespecífico, por exemplo, cirurgias prévias ou radioterapia ${ }^{2,3}$.

A presença de um tumor maligno pode levar a uma maior produção de mediadores inflamatórios, tais como: interleucina 1 (IL-1), interleucina 6 (IL-6), fator de necrose tumoral alfa (TNF- $\alpha$ ) e interferon gama (IFN- $\gamma$ ). A secreção excessiva e prolongada dessas citocinas traz efeitos adversos relacionados com a anorexia e a caquexia, com depleção dos estoques de tecido adiposo, degradação da massa magra e aumento da síntese de proteínas de fase aguda $^{2}$.

Outros fatores relacionados à desnutrição são a localização e estágio da doença. Nesse aspecto, pacientes com câncer avançado costumam apresentar pior estado nutricional ${ }^{2}$.

Aqueles diagnosticados com neoplasias do trato digestivo são, particularmente, mais susceptíveis à deterioração do estado nutricional. Além das anormalidades metabólicas já citadas, existe a redução da ingestão alimentar e/ou absorção de nutrientes em razão de sintomas gastrointestinais relacionados ao tumor, como náuseas, vômitos, diarreia ou disfagia ${ }^{4}$.

No tratamento quimioterápico, a ocorrência da desnutrição irá depender da frequência e do tempo que o paciente é submetido ao tratamento, da duração da manifestação dos sintomas e da adequação da ingestão alimentar ${ }^{5}$.

Pacientes que apresentam perda de peso durante a quimioterapia relatam maior toxicidade e sintomatologia do que aqueles que apresentam peso estável; por sua vez, a toxicidade das terapias pode ser exacerbada pela desnutrição ou perda de peso não intencional ${ }^{4}$, reduzindo a tolerância e a efetividade do tratamento antineoplásico e levando a uma maior morbimortalidade e redução da qualidade de vida ${ }^{3,5}$.

A Avaliação Subjetiva Global Produzida pelo Próprio Paciente (ASG-PPP) permite uma rápida avaliação do estado nutricional, identificação de sintomas de impacto nutricional, facilitando a implementação da terapia nutricional adequada ${ }^{6}$. A pontuação da ASG-PPP pode ser utilizada como medida de resultado da intervençáo nutricional e identifica mudanças sutis no estado nutricional, o que a torna uma ferramenta de elevada sensibilidade e especificidade ${ }^{6,7}$. É comum a existência de risco nutricional ou desnutrição em pacientes submetidos à quimioterapia ${ }^{8-13}$, entretanto o percentual de desnutrição pode variar dependendo do método de avaliaçáo.

Portanto, o objetivo do presente estudo foi descrever a classificação do estado nutricional, segundo método subjetivo, e os indicadores de risco nutricional no paciente oncológico em quimioterapia.

\section{MÉTODO}

Estudo descritivo, do tipo série de casos, envolvendo 30 pacientes, período de agosto a dezembro de 2014 , no setor de Quimioterapia do Hospital das Clínicas da Universidade Federal de Pernambuco (HC/UFPE). Foram elegíveis pacientes de ambos os sexos, com idade $\geq 20$ anos, diagnosticados com câncer, em acompanhamento ambulatorial e que haviam iniciado o primeiro ciclo de quimioterapia. Foram excluídos pacientes em cuidados paliativos, internados e com outras doenças crônicas consumptivas (nefropatas, hepatopatas, pacientes com Síndrome da Imunodeficiência Adquirida etc).

A pesquisa foi realizada após análise e aprovação do projeto pelo Comitê de Ética do Centro de Ciências da Saúde da UFPE (CEP/CCS/UFPE) sob o CAAE no 31173014.6.0000.5208, de acordo com a Resolução no 466/2012 sobre "Pesquisa envolvendo Seres Humanos", do Conselho de Saúde do Ministério da Saúde.

As variáveis socioeconômicas coletadas foram sexo, idade, nível de escolaridade, profissão e renda familiar mensal. O diagnóstico do tipo de neoplasia, a localização do tumor primário e o esquema quimioterápico utilizado corresponderam às variáveis clínicas coletadas das prescriçôes médicas e dos prontuários dos pacientes e registradas em formulário específico.

Segundo a localização do tumor e a terapia antineoplásica, os pacientes foram classificados em baixo risco, risco médio e alto risco nutricional. Foram considerados de baixo risco nutricional os indivíduos que recebiam esquemas quimioterápicos derivados da vinca, com baixas doses de metotrexato ou 5-fluoruracil (5-FU) em bolos e os diagnosticados com tumores musculoesqueléticos ou de próstata/mama ${ }^{14}$.

Pacientes que recebiam doses maiores que $80 \mathrm{mg} / \mathrm{m}^{2}$ de cisplatina a cada três semanas e antracíclicos (5-FU, irinotecano, docetaxel, ifosfamida, ciclofosfamida, decarbazina) ou que possuíam cânceres do colo uterino foram classificados de médio risco nutricional ${ }^{14}$. 
Foram considerados de alto risco nutricional os pacientes que tinham a quimioterapia e a radioterapia concomitantes, além dos diagnosticados com tumores malignos gastrointestinais ou hematológicos ${ }^{4,6}$.

Para avaliação do estado nutricional, utilizou-se a ASG-PPP na versão traduzida e validada por Gonzalez et al. ${ }^{15}$. A parte inicial do formulário contém perguntas sobre mudanças recentes no peso corporal, realização de atividades cotidianas, alteraçóes na ingestâo alimentar e sintomas que a influenciam e é preenchida com informaçóes geradas pelo participante da pesquisa. Quando o entrevistado era analfabeto ou encontrava dificuldade em responder ao formulário, o pesquisador auxiliava no preenchimento.

O restante do questionário foi respondido pelo pesquisador, com informaçóes sobre o diagnóstico do paciente, estresse metabólico e exame físico. Para categorização do estado nutricional, utilizaram-se os indicadores obtidos no formulário. A inclusão nas categorias A (bem nutrido), B (moderadamente desnutrido ou suspeito de desnutriçáo) ou C (gravemente desnutrido) foi realizada segundo a presença e gravidade dos sintomas apresentados por cada entrevistado.

A indicação de intervenção nutricional específica foi descrita de acordo com a pontuação obtida pelo escore numérico total da ASG-PPP ${ }^{15}$. Pontuação até 1 indica que não há necessidade de intervenção no momento; porém essa deve ser avaliada de forma rotineira durante o tratamento. Pontuaçóes de 2 a 3 indicam que o paciente e seus familiares devem ser educados por nutricionista ou outro profissional de saúde, com necessidade de intervenção farmacológica de acordo com os sintomas identificados pela ASG-PPP. De 4 a 8 pontos, há necessidade de intervenção pelo nutricionista, juntamente com médico e/ou enfermeiro como indicado pelo inquérito dos sintomas. Pontuaçáo igual ou superior a 9, indica necessidade crítica de melhora no manuseio dos sintomas e/ou opçóes de intervenção nutricional ${ }^{15}$.

Os pacientes que obtiveram pontuação a partir de 2 receberam encaminhamento para marcação de consulta no ambulatório de Nutrição/Oncologia do HC/UFPE.

\section{RESULTADOS E DISCUSSÃO}

A amostra foi composta por 30 pacientes com idade média de 49,1 $\pm 16,4$ anos. Cerca de um terço da amostra apresentou câncer do trato gastrointestinal (TGI) e a metade foi de pacientes em pós-operatório de cirurgias oncológicas: hemicolectomias, retossigmoidectomias, mastectomias, histerectomias, gastrectomias e amputaçôes. A quimioterapia apareceu como modalidade única de tratamento em 25 pacientes, os demais realizavam a quimioterapia em associação com a radioterapia. As características sociodemográficas e clínicas encontram-se na Tabela 1.

Segundo a localização do tumor e o esquema quimioterápico, $66,67 \%$ e $100 \%$, respectivamente, foram considerados de médio a alto risco nutricional

Tabela 1. Características socioeconômicas e clínicas de pacientes oncológicos em quimioterapia. Recife (PE), Brasil - 2014

\begin{tabular}{|c|c|c|}
\hline Características & Frequência & Porcentagem \\
\hline \multicolumn{3}{|l|}{ Sexo } \\
\hline Masculino & 15 & 50 \\
\hline Feminino & 15 & 50 \\
\hline \multicolumn{3}{|l|}{ Escolaridade } \\
\hline Ensino fundamental & 10 & 33,33 \\
\hline Ensino médio & 13 & 43,33 \\
\hline Ensino superior & 2 & 6,67 \\
\hline Não alfabetizado & 5 & 16,67 \\
\hline \multicolumn{3}{|l|}{ Renda } \\
\hline S 1 salário mínimo & 3 & 10,00 \\
\hline 2 a 3 salários mínimos & 23 & 76,67 \\
\hline > 3 salários mínimos & 4 & 13,33 \\
\hline \multicolumn{3}{|l|}{ Comorbidades } \\
\hline $\begin{array}{l}\text { Hipertensão arterial } \\
\text { sistêmica (HAS) }\end{array}$ & 4 & 13,33 \\
\hline Diabetes mellitus (DM) & 3 & 10,00 \\
\hline HAS e DM & 1 & 3,33 \\
\hline Sem comorbidades & 22 & 73,33 \\
\hline \multicolumn{3}{|l|}{ Localização do tumor } \\
\hline Sarcomas & 5 & 16,67 \\
\hline Hematológicos & 6 & 20,00 \\
\hline Próstata/Mama & 6 & 20,00 \\
\hline Colo do útero & 2 & 6,67 \\
\hline Trato gastrointestinal & 11 & 36,67 \\
\hline \multicolumn{3}{|l|}{ Presença de metástases } \\
\hline Sim & 9 & 75,00 \\
\hline Não & 3 & 25,00 \\
\hline \multicolumn{3}{|l|}{ Tipo de tratamento } \\
\hline Quimioterapia & 25 & 83,33 \\
\hline $\begin{array}{l}\text { Quimioterapia + } \\
\text { radioterapia }\end{array}$ & 5 & 16,67 \\
\hline \multicolumn{3}{|l|}{ Cirurgias } \\
\hline Sim & 15 & 50,00 \\
\hline Não & 15 & 50,00 \\
\hline \multicolumn{3}{|l|}{ Radioterapia } \\
\hline Sim & 5 & 16,67 \\
\hline Não & 25 & 83,33 \\
\hline \multicolumn{3}{|c|}{ Frequência da quimioterapia } \\
\hline Semanal & 5 & 16,67 \\
\hline Quinzenal & 8 & 26,67 \\
\hline Mensal & 15 & 50,00 \\
\hline Diária & 2 & 6,67 \\
\hline
\end{tabular}

$\mathrm{n}=30$ pacientes. Para a variável presença de metástase, $\mathrm{n}=12$ pacientes. 
(Tabela 2). Da mesma forma, o Inquérito Brasileiro de Nutrição Oncológica (IBNO) ${ }^{16}$ reúne resultados da ASG-PPP e mostra elevada frequência de desnutrição ou risco nutricional em pacientes com câncer do TGI, que apresenta maior impacto sobre os hábitos alimentares. Em pacientes com tumores de cavidade oral, esôfago e estômago, encontram-se percentuais de desnutrição ou risco nutricional que variaram de $62 \%$ a $84 \%$ dos pacientes. $\mathrm{O}$ mesmo inquérito verificou que, nas doenças hematológicas malignas, como leucemias e linfomas, também são elevados os percentuais de desnutrição ou presença de risco nutricional. Nos indivíduos com câncer de mama e próstata, esses percentuais são menores, $25 \%$ e $35,4 \%$, respectivamente. Pacientes sob alto risco nutricional, com base no tipo de tumor e no tratamento antineoplásico, têm indicação de terapia nutricional ${ }^{14}$.

Assim, parece que o uso da ASG-PPP torna viável a implementaçáo de um protocolo para pacientes em tratamento quimioterápico. Isso é relevante, uma vez que, na amostra estudada, $76,67 \%$ apresentaram comprometimento nutricional, sendo 17 (56,67\%) pacientes com risco nutricional ou desnutriçáo moderada (grau B) e seis (20\%) com desnutriçáo grave (grau C). Similarmente, outros estudos verificaram que é comum

Tabela 2. Risco nutricional segundo a localização do tumor e esquema quimioterápico. Recife (PE), Brasil - 2014

\begin{tabular}{l|c|c}
\hline $\begin{array}{c}\text { Indicadores de risco } \\
\text { nutricional }\end{array}$ & Frequência & Porcentagem \\
\hline \begin{tabular}{l|l} 
Localização do tumor \\
\hline Baixo risco nutricional
\end{tabular} & 10 & 33,33 \\
\hline Médio risco nutricional & 3 & 10,00 \\
\hline Alto risco nutricional & 17 & 56,67 \\
\hline Esquema quimioterápico & \\
\hline Baixo risco nutricional & 0 & 0 \\
\hline Médio risco nutricional & 26 & 86,67 \\
\hline Alto risco nutricional & 4 & 13,33 \\
\hline $\mathrm{n}=30$ pacientes. & &
\end{tabular}

a desnutrição no paciente oncológico em quimioterapia. Estudo descritivo envolvendo pacientes com câncer ginecológico encontrou $53,5 \%$ com desnutrição grave pela ASG-PPP, o percentual de desnutrição era maior naqueles que estavam em quimioterapia ${ }^{9}$. Em pacientes com câncer de cabeça e pescoço em quimioterapia, 38 $(59,5 \%)$ foram classificados como risco nutricional ou desnutrição (em grau B ou C) $)^{3}$. Utilizando a ASG-PPP, 27 (52\%) dos pacientes com câncer colorretal, antes de iniciarem radioterapia, foram classificados em grau $\mathrm{B}$ ou $\mathrm{C}^{10}$. Depressão e falta de apetite aparecem como fatores preditivos de desnutrição e devem ser considerados junto com a avaliação do estado nutricional ${ }^{9}$. Otimizar o estado nutricional e controlar os sintomas gastrointestinais são necessários durante todo o tratamento quimioterápico para melhorar os resultados clínicos e a qualidade de vida ${ }^{11}$.

Além das alteraçóes metabólicas próprias do câncer, o paciente oncológico sofre o impacto das complicaçôes relacionadas ao tratamento antineoplásico ${ }^{17}$. A quimioterapia pode trazer efeitos importantes que comprometem o estado nutricional, como anorexia, náusea, vômito e diarreia ${ }^{8}$. Estudo longitudinal envolvendo 68 pacientes com câncer do TGI superior verificou perda progressiva de peso após início do tratamento oncológico ${ }^{11}$.

Pelo escore numérico obtido na ASG-PPP, a necessidade crítica de intervenção nutricional (escore $\geq 9$ ) foi verificada em mais da metade da amostra estudada; contudo, quando se soma a isso aqueles com escore de 4 a 8 , o percentual total que indica a necessidade de intervenção nutricional é 76,66\% (Tabela 3).

Hill et al. ${ }^{4}$ encontraram forte correlação entre a pontuação da ASG-PPP e toxicidade da radioterapia para tratamento de câncer gastrointestinal. Zhang, Lu e Fang ${ }^{12}$, estudando pacientes com câncer gastrointestinal, encontraram 98\% com necessidade de intervenção nutricional segundo a ASG-PPP. Após a terapia nutricional necessária, 54\% da amostra apresentaram melhora dos sintomas ${ }^{12}$. Menores níveis de albumina sérica e maior

Tabela 3. Avaliação Subjetiva Global de pacientes oncológicos em quimioterapia. Recife (PE), Brasil - 2014

\begin{tabular}{l|c|c}
\multicolumn{1}{c}{ Classificação e escore da ASG-PPP } & Frequência & Porcentagem \\
\hline Grau ASG-PPP & 7 & 23,33 \\
\hline Bem nutrido (grau A) & 17 & 56,67 \\
\hline Desnutrição moderada ou suspeita de desnutrição (grau B) & 6 & 20,00 \\
\hline Desnutrição grave (grau C) & 1 & 3,33 \\
\hline Escore ASG-PPP & 7 & 23,33 \\
\hline 0-1: Não há necessidade de intervenção & 7 & 23,33 \\
\hline 2-3: Educação do paciente e seus familiares & 16 & 53,33 \\
\hline 4-8: Necessita intervenção nutricional & & \\
\hline$\geq$ 9: Necessidade crítica de intervenção nutricional & & \\
\hline
\end{tabular}

$\mathrm{n}=30$ pacientes. ASG-PPP=Avaliação Subjetiva Global Produzida pelo Paciente. 
relação proteína $\mathrm{C}$ reativa/albumina são relacionados a maiores escores e aos graus B e C da ASG-PPP 2,18 .

Questionados sobre a variação do peso durante as duas últimas semanas, apenas quatro entrevistados (13,33\%) relataram diminuição do peso (Tabela 4). Resultado divergente do encontrado pelo $\mathrm{IBNO}^{16}$, que revelou mais de $40 \%$ da amostra apresentando perda de peso não intencional.

Ao comparar a alimentação habitual com a ingestão alimentar do último mês, 14 pacientes $(46,47 \%)$ afirmaram estar se alimentando em menor quantidade devido a sintomas que impedissem a alimentação suficiente, sendo a náusea, a hiporexia e a xerostomia seus principais sintomas (Tabela 4). Na literatura científica, a hiporexia, náuseas e vômitos aparecem como queixas em até $80 \%$ dos pacientes em tratamento quimioterápico ${ }^{14,19,20}$. Gabrielson

Tabela 4. Peso corporal, ingestão alimentar e capacidade funcional de pacientes oncológicos em quimioterapia. Recife (PE), Brasil - 2014

\begin{tabular}{|c|c|c|}
\hline Características & Frequência & Porcentagem \\
\hline \multicolumn{3}{|l|}{ Peso corporal } \\
\hline Diminuição & 4 & 13,33 \\
\hline Manutenção & 20 & 66,67 \\
\hline Ganho & 6 & 20,00 \\
\hline \multicolumn{3}{|l|}{ Ingestão alimentar } \\
\hline S/mudanças & 13 & 43,33 \\
\hline Mais que o normal & 3 & 10,00 \\
\hline Menos que o normal & 14 & 46,47 \\
\hline \multicolumn{3}{|l|}{ Sintomas } \\
\hline $\begin{array}{l}\text { Sem problemas para } \\
\text { se alimentar }\end{array}$ & 10 & 33,33 \\
\hline $\begin{array}{l}\text { Alimentos têm sabor } \\
\text { estranho }\end{array}$ & 4 & 13,33 \\
\hline Xerostomia & 5 & 16,67 \\
\hline Hiporexia & 9 & 30,00 \\
\hline Náusea & 10 & 33,33 \\
\hline Constipação & 4 & 13,33 \\
\hline $\begin{array}{l}\text { Náuseas com odor } \\
\text { dos alimentos }\end{array}$ & 7 & 23,33 \\
\hline Saciedade precoce & 4 & 13,33 \\
\hline Mucosite & 2 & 6,67 \\
\hline Diarreia & 3 & 10,00 \\
\hline Problemas ao deglutir & 2 & 6,67 \\
\hline Dor & 1 & 3,33 \\
\hline Vômito & 1 & 3,33 \\
\hline \multicolumn{3}{|l|}{ Capacidade funcional } \\
\hline Normal & 5 & 16,67 \\
\hline $\begin{array}{l}\text { Em quase todas as } \\
\text { atividades }\end{array}$ & 4 & 13,33 \\
\hline $\begin{array}{l}\text { Na cama menos da } \\
\text { metade do dia }\end{array}$ & 10 & 33,33 \\
\hline Pouca atividade & 7 & 23,33 \\
\hline Acamado & 4 & 13,33 \\
\hline
\end{tabular}

et al. ${ }^{13}$, em pesquisa com 90 pacientes em quimioterapia, verificaram perda de apetite $(30 \%)$, alteração do paladar (31\%), fadiga (30\%) e diminuição da capacidade de realizar atividades do cotidiano em $53 \%$ da amostra. $\mathrm{O}$ aparecimento frequente de sintomas do TGI em pacientes recebendo quimioterapia se deve às drogas quimioterápicas administradas que atingem, além das células tumorais, as células de alta capacidade de replicação, como as da mucosa gastrointestinal ${ }^{19}$.

A toxicidade direta da droga principalmente, e seu impacto na medula óssea, causando neutropenia, são responsáveis pelas reações adversas na mucosa. A mais atingida é mucosa bucal por ser altamente sensível e desenvolver uma reação inflamatória em reposta aos efeitos tóxicos dos quimioterápicos. Pode ocorrer atrofia da mucosa da cavidade oral e infecção da mesma por fungos, bactérias ou vírus. Tais eventos descritos causam xerostomia, odinofagia e mucosite ${ }^{21,22}$.

A presença de náuseas e vômitos irá depender da dose e da droga prescrita, da velocidade e via de administração, número de ciclos recebidos e combinação com outros quimioterápicos ${ }^{23,24}$. Todos os agentes quimioterápicos possuem potencial ematogênico, de maior ou menor intensidade. Assim, os sintomas podem iniciar de poucas horas até 24 horas após a administração dos medicamentos, devido à continuidade dos efeitos metabólicos no Sistema Nervoso Central (SNC) e TGI. Os mecanismos que envolvem o aparecimento de náuseas e vômitos não estão completamente elucidados ${ }^{23}$.

Em relação à capacidade funcional, foi encontrado resultado semelhante ao de Gabrielson et al. ${ }^{13}$ no presente estudo, em que apenas cinco pacientes $(16,67 \%)$ eram capazes de realizar suas atividades normalmente após o início da quimioterapia, e dez indivíduos (33,33\%) relatavam capacidade funcional reduzida, ficando na cama quase a metade do dia. A fadiga relacionada ao câncer é um fator que diminui a capacidade funcional diária dos pacientes $^{24}$, sendo experimentada por $72 \%$ a $95 \%$ de todos os pacientes oncológicos durante e após o tratamento quimioterápico ${ }^{20}$.

\section{CONCLUSÃO}

O presente trabalho confirma os achados de outros estudos quanto à presença comum de risco nutricional ou desnutrição nessa população. Consequentemente, é grande a necessidade de intervenção nutricional. Um indicador importante de risco nutricional considera os parâmetros da ASG-PPP, método que coloca o julgamento sobre as variáveis: perda de peso, diminuída ingestão dietética, sintomas com impacto nutricional, perda de massas adiposa subcutânea e muscular, e capacidade 
funcional. Parâmetros isolados de risco nutricional, tais como: percentual de perda de peso, localização da doença, sintomas do TGI, percentual da ingestão alimentar, também são úteis durante a assistência nutricional ao paciente clínico, como em quimioterapia. Entretanto, a ASG-PPP é método recomendado e validado que deve ser utilizado em pacientes oncológicos. Sugere-se que os serviços de saúde, de acordo com suas especificidades e características, implementem um protocolo de assistência nutricional para a população em tratamento quimioterápico cuja intervenção se concentre em função da classificação do estado nutricional e do risco nutricional segundo o tipo de tumor e a terapia antineoplásica.

\section{CONTRIBUIÇÕES}

Ambas as autoras contribuíram efetivamente em todas as etapas do planejamento e redação do texto.

\section{Declaraçáo de Conflito de Interesses: Nada a Declarar.}

\section{REFERÊNCIAS}

1. Instituto Nacional de Câncer José Alencar Gomes da Silva. Estimativa 2014: incidência de câncer no Brasil. Rio de Janeiro: INCA; 2014.

2. Gomes de Lima KV, Maio R. Nutritional status, systemic inflammation and prognosis of patients with gastrointestinal cancer. Nutr Hosp. 2012;27(3):707-14.

3. Arribas L, Hurtós L, Milá R, Peiró IF. Factores pronósticos de desnutrición a partir de la valoración global subjetiva generada por el paciente (VGS-GP) en pacientes com cáncer de cabeza y cuello. Nutr Hosp. 2013;28(1):155-63.

4. Hill A, Kiss N, Hodgson B, Crowe TC, Walsh AD. Associations between nutritional status, weight loss, radiotherapy treatment toxicity and treatment outcomes in gastrointestinal cancer patients. Clin Nutr. 2011;30(1):92-8.

5. Dias MV, Barreto APM, Coelho SC, Ferreira FMB, Vieira GBS, Cláudio MM, et al. O grau de interferência dos sintomas gastrintestinais no estado nutricional do paciente com câncer em tratamento quimioterápico. Rev Bras Nutr Clin. 2006;21(3):211-18.

6. Leuenberger M, Kurmann S, Stanga Z. Nutritional screening tools in daily clinical practice: the focus on cancer. Support Care Cancer. 2010;18 Suppl 2:S17-27.

7. Gupta D, Vashi PG, Lammersfeld CA, Braun DP. Role of Nutritional Status in Predicting the Length of Stay in Cancer: A Systematic Review of the Epidemiological Literature. Ann Nutr Metab. 2011;59(2-4):96-106.

8. Prado CD, Campos JADB. Nutritional status of patients with gastrointestinal câncer receiving care in a public hospital, 2010-2011. Nutr Hosp. 2013;28(2):405-11.
9. Nho JH, Kim SR, Kwon YS. Depression and appetite: predictors of malnutrition in gynecologic cancer. Support Care Cancer. 2014;22(11):3081-88.

10. Faramarzi E, Mahdavi R, Mohammad-Zadeh M, Nasirimotlagh B. Validation of nutritional risk index method against patient-generated subjective global assessment in screening malnutrition in colorectal cancer patients. Chin J Cancer Res. 2013;25(5):544-8.

11. Grace E, Mohammed K, Shaw C, Whelan K, Andreyev J. Malnutrition and gastrointestinal (gi) symptoms in patients with upper-gi cancer [resumo]. Gut. 2014;63 Suppl 1:A104.

12. Zhang L, Lu Y, Fang Y. Nutritional status and related factors of patients with advanced gastrointestinal cancer. Br J Nutr. 2014;111(7):1239-44.

13. Gabrielson DK, Scaffidi D, Leung E, Stoyanoff L, Robinson J, Nisenbaum R, et al. Use of an abridged scored Patient-Generated Subjective Global Assessment (abPG-SGA) as a nutritional screening tool for cancer patients in an outpatient setting. Nutr Cancer. 2013;65(2):234-9.

14. Garófolo A. Nutrição clínica, funcional e preventiva aplicada à oncologia: teoria e prática profissional. Rio de Janeiro: Rubio; 2012. Capítulo 6, Métodos de triagem e avaliação nutricional aplicados à oncologia; p. 49-61.

15. Gonzalez MC, Borges LR, Silveira DH, Assunção MCF, Orlandi SP. Validação da versão em português da avaliação subjetiva global produzida pelo paciente. Rev Bras Nutr Clin 2010;25(2):102-8.

16. Instituto Nacional de Câncer José Alencar Gomes da Silva. Inquérito Brasileiro de Nutrição Oncológica. Rio de Janeiro: Inca; 2013.

17. Coronha AL, Camilo ME, Ravasco P. The relevance of body composition in cancer patients: what is the evidence? Acta Med Port. 2011;24 Suppl 4:769-78.

18. Phippen NT, Lowery WJ, Barnett JC, Hall LA, Landt C, Leath CA 3rd.Evaluation of the Patient-Generated Subjective Global Assessment (PG-SGA) as a predictor of febrile neutropenia in gynecologic cancer patients receiving combination chemotherapy: a pilot study. Gynecol Oncol. 2011;123(2):360-4.

19. Calixto-Lima L, Gomes AP, Geller M, Siqueira-Batista, R. Dietetic management in gastrointestinal complications from antimalignant chemotherapy. Nutr Hosp. 2012;27(1):65-75.

20. Mota DDCF, Pimenta CAM. Fadiga em pacientes com câncer avançado: conceito, avaliação e intervenção. Rev Bras Cancerol. 2002;48(4):577-83.

21. Sanches Junior JA, Brandt HRC, Moure ERD, Pereira GLS, Criado PR. Reações tegumentares adversas 
relacionadas aos agentes antineoplásicos - Parte I. An Bras Dermatol. 2010;85(4):425-37.

22. Hespanhol FL, Tinoco EMB, Teixeira HGC, Falabella MEV, Assis NMSP. Manifestações bucais em pacientes submetidos à quimioterapia. Cien saúde coletiva. 2010;15 Suppl 1:1085-94.
23. Gozzo TO, Moyses AMB, Silva PR, Almeida AM. Náuseas, vômitos e qualidade de vida de mulheres com câncer de mama em tratamento quimioterápico. Rev Gaúcha Enferm. 2013;34(3):110-16.

24. Campos MPO, Hassan BJ, Riechelmann R, Giglio A. Fadiga relacionada ao câncer: uma revisão. Rev Assoc Med Bras. 2011;57(2):211-19. 


\section{Abstract}

Introduction: The Subjective Global Assessment produced by the patient is recommended for during the nutritional care in oncology, for the early detection of nutritional risk or malnutrition. The location of the tumor and the chemotherapy course may, in themselves, aid in the detection of nutritional risk. Objective: To describe the classification of nutritional status according to the subjective method, together with nutritional risk factors in cancer patients undergoing chemotherapy. Method: This is a case series study held at the chemotherapy unit of a university hospital, from August to December 2014. The participants were adult and elderly outpatients of both sexes, aged $\geq 20$ years, diagnosed with cancer, and who had begun the first cycle of chemotherapy. The location of the tumor and the antineoplastic therapy were used as indicators of nutritional risk. Nutritional assessment was performed via the Subjective Global Assessment produced by the patient themselves. Results: 30 patients were studied, with an average age of $49.1 \pm 16.4$ years. Twenty-five respondents (83\%) were treated solely with chemotherapy. According to the location of the tumor and the course of chemotherapy, $67 \%$ and $100 \%$ of the cases, respectively, were classified as medium to high nutritional risk. By subjective assessment, 17 (56.7\%) presented a nutritional risk or moderate malnutrition (Grade B) and 6 (20\%) severe malnutrition (Grade C). By way of the score via subjective assessment, the need for nutritional intervention was detected in 23 cases (76.7\%). Conclusion: The sample studied revealed high frequencies of tumors and aggressive therapies that could cause nutritional risk/malnutrition, highlighting the necessity of nutritional therapy.

Key words: Nutrition Assessment; Nutritional Status; Drug Therapy; Drug-Related Side Effects and Adverse Reactions; Neoplasms

\section{Resumen}

Introducción: La Valoración Subjetiva Global Generada por el Paciente es recomendada durante la asistencia nutricional en oncología para la detección precoz del riesgo nutricional o desnutrición. La localización del tumor y del esquema quimioterápico pueden por si solo auxiliar en la detección del riesgo nutricional. Objetivo: Describir la clasificación del estado nutricional según el método subjetivo y los indicadores de riesgo nutricional en el paciente con cáncer en quimioterapia. Método: Estudio del tipo serie de casos, realizado en el sector de quimioterapia de un hospital universitario, de agosto a diciembre de 2014. Participaron de la investigación individuos adultos y ancianos, de ambos los sexos, con edad $\geq 20$ ańos, diagnosticados con cáncer, acompañados ambulatoriamente y que habían iniciado el primer ciclo de quimioterapia. La localización del tumor y la terapia antineoplásica fueron utilizadas como indicadores de riesgo nutricional. La evaluación nutricional fue realizada por la Valoración Subjetiva Global Generada por el Paciente. Resultados: Fueron estudiados 30 pacientes, con edad media de 49,1 $\pm 16,4$ ańos. 25 encuestados (83\%) fueron tratados únicamente con quimioterapia. Segundo la localización del tumor y el esquema quimioterápico, 67\% y $100 \%$, respectivamente, fueron considerados de medio a alto riesgo nutricional. A través de la evaluación subjetiva, 17 (56,7\%) presentaron riesgo nutricional o desnutrición moderada (Grado B) y 6 (20\%) desnutrición grave (Grado C). A través de la puntuación obtenida del formulario subjetivo, la necesidad de intervención nutricional fue verificada en $23(76,7 \%)$ pacientes. Conclusión: En la muestra estudiada fueron altas las frecuencias de tumores y terapias agresivas de riesgo nutricional/desnutrición, así como se observó la necesidad de indicación de terapia nutricional. Palabras clave: Evaluación Nutricional; Estado Nutricional; Quimioterapia; Efectos Colaterales y Reacciones Adversas Relacionados con Medicamentos; Neoplasias 